\title{
Editorial: Vol 2, No 3 (2010)
}

The Online Journal of Public Health Informatics (OJPHI) was developed in recognition of the fact that, up until December, 2009, there was no journal dedicated to the dissemination of information about best informatics practices among public health practitioners, researchers, and educators. The OJPHI has attracted over 200 registered researchers, educators, policy makers, and practitioners from major universities, research institutes, and health care and public health agencies. Our current record indicates that the journal has a bright future as exemplified by the quality of the papers submitted for publication. The current issue contains interesting papers on topics ranging from the Infoshare, Crowdsourcing, to the application of Twitter to crisis management.

In the introductory article, entitled, "InfoShare: An Information Sharing Tool for Public Health During the 2009 Presidential Inauguration and H1N1 Outbreak" the authors develop real-time electronic disease surveillance system for monitoring the health of a population during mass gatherings or special events covering multiple jurisdictions. Major public events with attendees from multiple regions, such as the Super Bowl, the Olympics, and Presidential Inaugurations, are examples of events that can benefit from cross-jurisdictional electronic surveillance to assess the health risks for the gathered and returning populations.

In the next paper, entitled" Utility of the ESSENCE Surveillance System in Monitoring the H1N1 Outbreak" the authors compare emergency department visits for Influenza-Like Illness from 2008 to 2009 in the National Capital Region. Information generated from the monitoring process enabled local public health practitioners to have an enhanced understanding of the magnitude of different ILI outbreaks in their jurisdictions, leading to the development of improved response and control measures for the novel 2009 H1N1 influenza outbreak.

The next paper entitled "Agent Based Modeling of 'Crowdinforming' as a Means of Load Balancing at Emergency Departments" develops a framework for modeling the outbreak of Influenza-Like Illness (ILI) with accompanying surges in hospital emergency departments. The method presented in this paper employs "Crowdinforming", a component of "Crowdsourcing" technology, to disseminate collected and processed information to the "crowd" via public access. The aim of this technology is to provide the public with reliable information for improved decision making about their visits to emergency departments during disease outbreaks.

Advances in information technology and software development have made it cost-effective to provide powerful and flexible analytic capability to local public health units. In the paper entitled "NC CATCH: Advancing Public Health Analytics" the authors develop a Web-based analytical system for local public health units and their community partners in North Carolina. The system provides the public health units the ability to engage in outcomes-based performance 
measurements at the local level and the information required to develop appropriate programs to improve community health.

In recent years several public health educational institutions and government agencies have developed Web-based training programs for first responders and others likely to be involved in the management emergency situations. In general, the methodologies for evaluating the success of these online programs have been based on the traditional face-to-face models. In the final peer-reviewed paper of this issue, Dr.Priya Nambisan develops a methodology for evaluating online emergency preparedness courses that draws on theories of human computer interaction, distance learning, usability research, and online consumer behavior. The study outlines strategies for improving participants' pragmatic, hedonic, sociability, and usability experiences in an online technological environment.

In the final paper of this issue, published in the Working Papers Section of the Journal, Dr. Lisa Gualtieri of Tufts University School of Medicine describes the use communication channels ranging from Twitter, text messages to megaphones, to provide information to the citizens of Greater Boston when a major water pipe break interrupted water services. The emergency planning processes, the response strategies, and the lessons learned in this crisis are presented in this paper.

This is the final issue (Volume 2, Issue 3) of the Online Journal of Public Health Informatics in 2010. By all indications, this has been a challenging but worthwhile exercise. Many thanks to the editors, the journal manager, and all the volunteers who have been working so tirelessly to make this project a success. Through your hard work, the field of public health informatics now has a journal solely dedicated to the dissemination of information about best practices and latest research to our stakeholders.

Happy Holidays!!

Edward Mensah, $\mathrm{PhD}$

Editor-in-Chief

Online Journal of Public Health Informatics

1603 W Taylor St, Rm 757

Chicago. IL. 60612

Email: dehasnem@uic.edu

Office: (312) 996-3001 